XV.

Mittheilung aus der I. chirurgischen Universitätsklinik in Budapest (Director: Prof. Dr. Julius Dollinger).

\title{
Der Ureterenkatheterismus im Dienste einiger neuerer Methoden der Nierendiagnostik.
}

\author{
Von \\ Dr. Géza v. Illyés, \\ klinischer Assistent.
}

Bei der Diagnose der chirurgischen Erkrankungen der Niere spielen der Ureterkatheterismus und die gesonderte Untersuchung des Secretes der einzelnen Niere eine grosse Rolle, einerseits um festzustellen, welches die erkrankte Niere, andererseits um zu erfahren, ob die zweite Niere functionell intact ist, ob und in welchen Maasse sie die Function der anderen Niere übernommen hat.

Aus der Untersuchung des Gesammtharns beider Nieren können wir nicht erfahren, in welcher Weise sich die eine oder andere Niere an der Secretion betheiligt, ob bei Vorhandensein einer kranken Niere die gesunde sämmtliche Harnbestandtheile oder nur einen Theil derselben ausscheidet oder sich in der Function mit der kranken Niere, deren Functionsfähigkeit eine herabgesetzte ist, theilt. Vor der Operation ist die Feststellung dieser Thatsache um so wichtiger, als es Fälle giebt, wo zwei pathologische Nieren im Stande sind, sämmtliche Harnbestandtheile aus dem Organismus auszuscheiden, wenn wir jedoch die eine Niere durch Operation aus der Function ausschliessen, so tritt Insufficienz mit allen ihren Folgen ein. Wenn nur eine Niere pathologisch ist, so übernimmt die normale Niere langsam die herabgesetzte Function der erkrankten.

Dies ist der Grund, weshalb es vor dem operativen Eingriff nicht genügt, zu wissen, dass eine Nierenerkrankung vorliegt, welche wir auf operativem Wege behandeln wollen, sondern ebenso wichtig ist die Feststellung dessen, ob die andere Niere functionell intact ist, die Function der kranken Niere übernommen hat, und in welchem Maasse dieselbe im Stande ist, selbständig zu functioniren. 
Die Feststellung dieser Thatsache ist möglich, wenn wir im Stande sind, den Harn sowohl der einen als auch der anderen Niere gesondert aufzufangen und zum Gegenstand der Untersuchung zu machen.

Das gesonderte Auffangen des Harns der zwei oder zu mindest der einen Niere beschäftigt schon seit langer Zeit die Aerzte.

Tuchmann war der Erste, der ein lithotriptorartiges Instrument construirte, mit welchem er nach Einfuihrung in die Blase die eine Ureteröffnung abklemmte und den in die Blase träufelnden Harn der anderen Niere auffing.

Ebermann hat ein ähnliches Instrument construirt, deren eine Branche in der Blase, die andere im Rectum lag.

Silbermann wollte mittelst einer mit Quecksilber geftullten Gummirohrs die eine Ureteröffnung comprimiren.

Hegar und Sänger empfehlen in jenen Fällen, wo der Creterverlauf durch die Vagina getastet werden kann, denselben an dieser Stelle zu suchen, za umstechen und für eine Zeit lang sein Lumen in dieser Weise zu verschliessen.

E. H. Fenwick, der ein katheterartiges Instrument in die Blase einfuhrte, hat die auf der einen Seite desselben befindliche Oeffnung auf die Vretermündung gelegt, angedrückt und mittelst eines Aspirationsballons eine gewisse Quantität Harn aus dem Ureter herausgepumpt.

Der Neumann'sche sogenannte Harnscheider ist ein in der Mitte zweigetheiltes Instrument, welches wir in die Blase einführen. Die hintere Blasenwand wird hierauf mit dem in die Scheide eingefthrten Finger an das Instrument angedrückt; der aus den beiderseitigen Ureteren abträufelnde Harn fliesst sowohl auf der einen wie auf der anderen Seite des Instrumentes herab und wird in abgetheilten Eprouvetten aufgefangen. Von diesen Verfahren haben wir Letzteres an unserer Klinik in mehreren Fällen versucht. Bei einseitiger Pyonephrose gelang es, eitrigen Harn gesondert aufzufangen, jedoch beim Andrücken der Blasenwand an das Instrument erlitt dieselbe eine Quetschung und begann zu bluten und zn schmerzen. In anderen Fällen gelang die Absonderung des Harnes nicht. Das Verfahren ist daher nicht so verlässlich, um aus demselben exacte Folgerungen ableiten zu können. Die übrigen Methoden können wir nicht mehr anwenden. Durch Katheterisation des Ureters sind wir im Stande, längere Zeit hindurch den Harn der betreffenden Niere gesondert aufzufangen.

In früheren Zeiten hat man sich einen besonderen Weg zur Uretermündung gebahnt. So eröffnete $\mathrm{E} \mathrm{mm}$ et die Blase von der Scheide aus, Bozemann legte gleichfalls eine breite Blasenscheidenfistel behufs Ureterkatheterisation an. Harrison empfiehlt bei Männern den Perinealschnitt oder, wenn die grosse Prostata ein Hinảerniss abgiebt, den hoben Blasenschnitt. Guyon und Albarran haben zu gleichem Zwecke die Epicystotomie ausgeführt. Heutzutage wird der Ureterenkatheterismus sowohl bei Männern als auch bei Frauen auf natürlichem Wege vorgenommen. Wir kennen dreierlei Verfahren: 1) Ohne Endoscop und 
Cystoskop; 2) mit Endoscop bei reflectirtem Lichte; 3) mit Cystoskop bei directer Beleuchtung.

Simon war der erste, der in der Weise vorging, dass er nach Erweiterung der weiblichen Urethra mit den Fingern in die Blase eindrang und unter Leitung desselben einen geknöpften Metallkatheter in die Uretermündung einführte.

Pawlick beschrieb im Jahre 1886 sein Verfahren, welches darin besteht, dass ein Katheter ohne vorherige Dilatation der Urethra in die Blase und entlang ihrer hinteren Wand eingeführt, dann ein wenig seitwärts gedreht wird; unter langsamen Vorschieben gelangt derselbe in den Ureter. Wird das Instrument jetzt losgelassen, so verharrt es in seiner Lage, während dasselbe sofort herausfällt, falls es sich nicht im Ureter befindet. In mehreren Fällen gelang $\mathrm{Paw}$ ack die Finfuhrung des Katheters, trotzdem fand das Verfahren keine Verbreitung, weil seine Ausführung mit Schwierigkeiten verbunden ist und viel Uebung erfordert.

Den Katheterismus mit Hilfe des Endoskops beschrieb der NewYorker Arzt Kelly.

Patientin befindet sich in Steinschnitt oder Knieellbogenlage. Nacb Dilatation der Urethra wird ein kleines mit abgerundetem Mandrin versehenes Metallrohr von $10-15 \mathrm{~mm}$ Durchmesser in die Blase eingeführt. Nach Entfernung des Mandrins fliesst der in der Blase befindliche Harn heraus und Luft dringt ein, welche die Blasenwände ausdehnt. Mittelst elektrischem Stirnreflector wird nun das Blaseninnere beleuchtet und die Uretermiindung aufgesucht, in der Weise, dass das Instrument bis zur inneren Blasenmündung zurückgezogen, beiläufig $30^{0}$ seitwärts gewendet und einige Centimeter weit vorgeschoben wird. Das eingestellte Gebiet wird mit einer langen geknöpften Sonde abgetastet und nach Auffinden der Uretermündung, statt der Sonde ein Katheter in dieselbe eingefithrt. Das Verfahren ist allenfalls leichter als die vorherigen, obzwar der ausfliessende Harn, sowie der Umstand, dass die eindringende Luft nicht immer die Falten der Blasenwand ausgleicht, häufig störend sind. Besonders wenn Blasenkatarrh und Schwellung der Schleimhaut vorhanden ist. Ferner ist auch die gehörige Dilatation der Urethra mit Schmerzen verbunden; weshalb wir das Verfahren zu Ureterkatheterisationszweckeu weniger verwenden. Auf unserer Klinik habe $\mathrm{ich}$ in einem Falle mittelst Kelly'schem Spiegel die locale Behandlung von tuberculösen Blasengeschwären vorgenommen, welchen Zwecken das Instrument vollkommen entspricht.

Ein ähnliches Verfahren beschreibt Morris, der beim Manne oder Weibe verschieden lange am Ende schief abgeschnittene und abgestumpfte mit Mandrin versehene Metallröhren durch die Urethra in die Blase einführt; nach Entfernung des Mandrins dringt Luft ein, die Beleuchtung geschieht mittelst Stirnreflectors. Die hintere untere Blasenwand wird mit dem, beim Weibe in die Vagina beim Manne in das Rectum, eingeführten Finger empor gehoben. Die Blasenwand kann unter langsamem Vorziehen des Instrumentes übersehen und der Ureter nach entsprechender Einstellung katheterisirt werden.

Allgemeiner Anwendung erfreut sich derzeit das Sondiren der Ureteren 
mit dem Cystoskop, bei directer Beleuchtung, welches sowohl beim Manne als auch beim Weibe ausfuhrbar ist.

Brenner war der erste, der an der convexen Seite des Cystoskops einen Kanal anlegte, durch den er einen Katheter in die cystoskopisch eingestellte Uretermündung einfühte ; in ähnlicher Weise verfuhr B o i s s e a u du Rocher mit Hilfe seines Megaloskops. Mit diesem gelang der Ureterenkatheterismus beim Weibe, Poirier sogar beim Manne.

Bei den neueren Cystoskopen verläuft der Kanal zur Aufnahme des Katheters an der concaven Seite des Instrumentes, wo sich auch Prisma und Lampe befinden.

Beim Casper'schen Cystoskop liegt das Linsensystem nicht in einer Linie, sondern der Schaft des Instrumentes ist bajonettförmig abgeknickt, was nach Casper deshalb vortheilhaft ist, weil der Ureterenkatheterismus leichter von statten geht, wenn der Schaft nicht im Wege ist. Das diese Brechung des. Bildes hervorbringende Prismensystem trägt jedoch auch dazu bei, dass das in der Blase gesehene Bild nicht hell genug, nicht rein genng ist. Die Beleuchtung ist eine schwache, und auch die Handhabung des Instrumentes eine schwere, so dass $\mathrm{C}$ as per selbst behauptet, dass der Katheterismus mit Hilfe desselben nicht immer gelingt.

Vollkommen sind die von Nitze und Albarran construirten Uretercystoskope.

Das Nitze'sche Ureterencystoskop hat einen Umfang von 27 Charrière. Der zur Aufnahme des Katheters bestimmte Kanal verläuft auf der concaven Seite; am vesicalen Ende des Kanals befindet sich eine kleine Platte, welche mittelst einer am Instrumente befindlichen Schraube in verschiedenen Winkeln eingestellt werden kann und welche die Richtung des aus dem Kanal heraustretenden Katheters näher bestimmt. Die kleine Platte lässt sich jedoch nicht ganz horizontal legen, sondern steht unter einem gewissen Winkel, was beim Vorschieben dünerer Katheter ein wesentliches Hinderniss abgeben kann, besonders wenn sich der Katheter durch die Fltissigkeit ein wenig erweicht. Ein weiterer Nachtheil des Instrumentes besteht darin, dass sich die vesicale Mündung des Kanals allzuweit vom Prisma befindet, so dass der austretende Katheter $24 \mathrm{~mm}$ weit herauskommen muss, damit seine Spitze sichtbar werde; der aus weichem Material verfertigten Katheter kann jedoch nur solange dirigirt werden, als er in einem Kanal mit fester Wandung liegt; ein je grösseres Stück desselben von dort austritt, um so weniger sind wir im Stande, demselben eine Richtung zu geben; daher kommt es, dass die Falten der Blasenschleimhaut den weichen Katheter zuweilen ablenken. Diese grosse Entfernung der Kanalmündung vom Prisma ist auch die Ursache dessen, warum in gewissen Fällen, wo die Uretermündung näber zum Orificium internum liegt, die Katheterisation dieses Ureters mit dem Instrumente schwer oder tiberhaupt nicht gelingt, und zwar deshalb nicht, weil nach Einstellung des Prismas vor den Ureter der aus dem Kanal austretende Katheter sich entweder vollkommen in der Schleimhaut des Orificium vesicae internum verfängt oder aber die Schleimbaut ritzt, wo dann die sich einstellende Blutung änsserst störend wirkt.

Das Linsensystem des Instrumentes ist jedoch ein ausgezeichnetes, 
das Gesichtsfeld sehr gross, die Belenchtung eine vollkommene. Das cystoskopische Bila also schön rein.

Das Albarran'sche Uretercystoskop hat einen Umfang von 25 Charrière; auch hier ist die am Ende des Kanals befindliche Platte angebracht, welche jedoch auch horizontal gelegt werden kann, und da dieselbe ohnehin innerhalb weiterer Grenzen beweglich ist, so steht sie dem austretenden Katheter nicht im Wege. Die Kanalöffnung liegt näber zum Prisma, weshalb ein kleineres Stiick des Katheters austreten muss, damit es gesehen und demselben die nöthige Richtung gegeben werden könne. Diese Umstände erleichtern die Ureterkatheterisation mit dem Instrumente.

Der äussere Theil des Kanales zerfällt in zwei Theile, der eine dient zur Aufnahme des Katheters, der andere durch Schraubenvorrichtung versehliessbare Theil dazu, um durch denselben die Flitssigkeit, falls dieselbe trubbe oder nndurchsichtig geworden, abfiessen, klare Flüssigkeit einfliessen zu lassen, das Prisma von eventuellen Verunreinigungen, Schleim, Blut, mit einem Strahl befreien zu können. Die Beleuchtung ist jedoch eine weniger vollkommene, da ein grosser Theil der Mignonlampe in der am Katheterrande befindlichen Metallhülse liegt, auch das Linsensystem ist kein solch gutes, das cystoskopische Bild kein solch reines, das Sehfeld nicht so gross.

Vor Ausführung der Katheterisation müssen Instrument und Katheter auf das Genaueste sterilisirt werden; die Sterilisation kann in Albarran'schen Thermoformogen Schrank geschehen oder aber durch Einlegen in 5 proc. Carbolsäure seine halbe Stunde vor dem Gebrauche, mehrmaliges Durchspritzen mit der Lösung und schliesslich energisches Abreiben.

Es griebt Cystoskope, welche nach dichtem Verschluss des am äusseren Theile des Instrumentes befindlichen Linsensystems mittest Stöpsels, angeblich auch durch Auskochen sterilisirt werden können. Ich machte jedoch die Erfahrung, dass sich die Linsen nach dem Auskochen zu Folge Schmelzens der fixirenden Substanz bis zur Unbrauchbarkeit des Instrumentes trüben.

Hierauf werden die Hände sowie der zu untersuchende Patient gewaschen. Vorbedingungen der Ausführbarkeit der Untersuchung sind, dass die Urethra für Charrière 25 durehgängig sei und die Blase eine Capacität von 50-200 g Fliissigkeit habe, schliesslich dass die in der Blase befindliche Flüssigkeit rein und durchsichtig sei. Nach Reinigung des Orificium externum wird die Blase gewaschen; am zweckmässigsten ist "es, dieselbe mit $150 \mathrm{~g} 4$ proc. Borlösung zu füllen.

Tach Einschieben des Ureterkatheters in den Metallkanal wird das Instrument mit Glycerin schlüpfrig gemacht und in derselben Weise wie ein Metallkatheter in die Blase eingeführt. 
Jetzt suchen wir im beleucliteten Blaseninnern die Ureterenmündungen auf.

$\mathrm{Zu}$ diesem Behufe wird das Instrument mit nach unten gerichtetem Prisma bis zum Orificium internum zurückgezogen, hierauf $2-3 \mathrm{~cm}$ weit vorgeschoben, bis das die zwei Harnleitermündungen verbindende Ligamentum interuretericum als etwas erhabene Falte ins Auge fällt. Diesem entlang wird das Instrument beiläufig $30^{\circ}$ nach rechts oder links gewendet, bis die spaltförmige Harnleitermündung im Gesichtsfelde erscheint und der aus derselben in Intervallen ausströmende trübe oder reine Harnstrahl sichtbar wird.

Nach Einstellung der Harnleitermündung wird das Instrument in dieser Lage mit der einen Hand fixirt und der Ureterkatheter mit der anderen, der Seite des Ureters entsprechenden Hand langsam vorgeschoben, bis die Katheterspitze vor dem Prisma zum Vorschein kommt; jetzt muss letztere entweder durch Seitwärtswenden des ganzen Instrumentes oder mit Hülfe der kleinen Platte so gerichtet werden, dass dieselbe in die Uretermündung gelangt. Das ist der schwierigste Theil des ganzen Verfahrens, besonders in einzelnen ungünstigen Fällen, denn wenn die Katheterspitze einmal in der Ureterenmïndung darin ist, so gelingt das langsame Vorschieben - vorausgesetzt, dass im Ureter kein Hinderniss besteht - sehr leicht bis hinauf ins Nierenbecken, was wir daran sehen können, dass der vesicale Theil des Instrumentes nicht tiefer in den Ureter dringt, sondern sich in der Blase krümmt.

Jetzt wird das Glühlämpchen des Cystoskops verlöscht, und indem wir mit der einen Hand den Katheter im Kanal fortwährend vorschieben, ziehen wir mit der andern das Instrument langsam aus der Blase heraus; beim Orificium externum angelangt, wird der aus dem Kanal austretende Ureterkatheter fixirt und das Instrument ganz entfernt.

Die Fixation des Harnleiterkatheters geschieht am zweckmässigsten in der Weise, dass derselbe an der Harnröhrenmündung mit einem Faden umbunden, letzterer beim Weibe an die Schamhare, beim Manne mittelst Heftpflasterstreifen an den Penis befestigt wird.

Den aus der einen Niere durch den Katheter austropfenden Harn können wir in beliebiger Menge sammeln. Der Harn der anderen Niere wird in der Weise aufgefangen, dass in die Blase ein Dauerkatheter eingeführt wird, durch welchen der Gesammtharn dieser Niere ausfliesst, oder es wird in den anderen Ureter gleichfalls ein Katheter eingelegt. - Der gesammelte Harn kann auf verschiedene Weise untersucht werden. Die eine Methode ist die chemische 
Untersuchung des Harns. Es giebt jedoch Nierenerkrankungen, wo die chemische Constitution des Harns kaum eine Veränderung erfährt z. B. bei Neoplasmen, den solitären corticalen Formen der Nierentuberculose, Steinbildung im Nierenbecken u. s. w., wo wir daher mit Hülfe dieser Untersuchungsmethode wenig über den Zustand der Nieren oder gar der einzelnen Niere erfahren. Die Bestimmung des Nitrogens und der Chloride giebt gleichfalls kein genaues Resultat, weil die Tagesschwankung derselben auch unter normalen Verhältnissen schon eine grosse ist. Von einer eimmaligen Bestimmung können wir daher nicht wissen, ob wir es mit einer Schwankung innerhalb normaler Grenzen oder einer geringen Ausscheidung zu thun haben; aus der Vergleichung des Secretes der zwei Nieren können wir jedoch Schlüsse von relativem Werthe ableiten. - Nur wenn grosse Abweichungen oder Schwankungen in der chemischen Constitution des Harns der einen oder anderen Niere bestehen, dann folgern wir aus dem Vergleiche darauf, dass die eine oder andere Niere insufficient ist; bei dieser Untersuchung erhalten wir daher keine absoluten, höchstens relative Werthe. In den von mir beobachteten Fällen hatte Herr Dr. Kövesi die Güte, die Bestimmung der Chloride und des $\mathrm{N}$. vorzunehmen.

Die zweite Methode ist die Prüfung der Durchgängigkeit der Niere mittelst Methylenblau. Nach $\Lambda$ chard und Castaigne wird dem Pat. eine Pravaz'sche Spritze voll von einer 5 proc. Methylenblaulösung steril subcutan injicirt und von da ab der Harn stïndlich gesammelt; unter normalen Verhältnissen erscheint das Methylenblau bereits in der ersten Stunde, fürbt den Harn blau und nach einigen Stunden erreicht die Ausscheidung ihr Maximum, um nach beiläufig 36-48 Stunden gänzlich zu verschwinden. Ein Theil des Methylenblau erscheint in reducirter Form als sogenanntes Chromogen, welches farblos ist, jedoch nach Ansäuern der zu untersuchenden Flüssigkeit mit einigen Tropfen Essigsäure und Aufkochen derselben in blauer Farbe erscheint; dasselbe wird während derselben Zeit ausgesehieden wie die vorerwähnte Form, nur in geringerer Menge.

Die Menge des ausgeschiedenen Methylenblau wird in der Weise bestimmt, dass wir eine Farbenscala von 1-24 anlegen, bei welcher Nr. 1 der Lösung eines Theiles Methylenblau in Million Theilen Wasser, Nr. 24 der von einem Theil Methylenblau in zehntausend Theilen Wasser entspricht. Mit dieser Farbenscala wird der durch das ausgeschiedene Methylenblau blaugefärbte Harn verglichen und die Intensität der Ausscheidung bestimmt. Die Bestimmung des Chromogen geschieht aus der Differenz in der Abweichung der an- 
gesäuerten und aufgekochten Lösung von der früher festgestellten Farbenscala.

Unter pathologischen Verhältnissen beginnt die Ausscheidung später, zieht sich lange hin, hört erentl. für einige Zeit auf, fängt wieder an und geht mit geringerer Intensität von Statten.

Bei der Untersuchung muss daher die Intensität der Ausscheidung in Betracht gezogen werden, denn eine durchgängige Niere lässt das Methylenblau in grösserer, eine weniger durchgängige in geringer Menge durch.

Ferner darf die Ausscheidungsdauer nicht ausser Acht gelassen werden; nach Albarran und Bernard ist eine protrahirte Ausscheidung in den meisten Fällen das Functionsresultat compensatorischer Hypertrophien; so erklären sie die lange Ausscheidungsdauer bei interstitieller Nephritis.

Schliesslich muss auch der Ablauf der Ausscheidung berücksichtigt werden, welche eine continuirliche, intermittirende, cyclische oder polycyclisehe sein kann.

Die Untersuchungsmethode ist sehr einfach, absolut ungefährlich jedoch nicht exact, da dieselbe nicht immer genau den Grad der Ausscheidung anzeigt, so dass Grocco Fälle beschreibt, in welchen die Pat. urämisch zu Grunde gingen, ohne dass die Ausscheidung: des Methylenblau eine wesentliche Abweichung von der Norm gezeigt häłte: die Methode giebt keinen sicheren Aufschluss über die anatomischen Veränderungen der Nieren, sondern zeigt nur die functionellen Abweichungen. Da die Bestimmung der erhaltenen Werthe mit Hülfe der Farbenscala minder genaue Resultate ergiebt, und so können wir aus einer normalen Ausscheidung nicht mit absoluter Genauigkeit auf anatomisch gesunde Nieren und vice versa aus einer abnormen Ausscheidung auf anatomisch kranke Nieren schliessen. Die Ursache kann auch in Functionsstörungen der Niere gelegen sein.

Diese Untersuchungsmethode kann daher auch bei einseitigen Erkrankungen der Niere nützliche Aufschliissse geben, jedoch nur bei gleichzeitiger Anwendung des Harnleiterkatheters.

Eine genaue Untersuchungsmethode ist die Bestimmung des Gefrierpunkts von Blut und Harn, die sogenannte Kryoskopie, welche wir auf Grundlage der Studien von Professor Alexander v. Koranyi kennen. Wenn die Niere den Harn aus dem Blute ausscheidet, so bringt sie zwischen den zwei Flïssigkeiten eine mächtige osmotische Druckdifferenz hervor, $d$. h. die moleculare Concentration wird in der einen grösser, in der anderen kleiner, welche wir in Zahlen a usdrücken können, indem wir den Gefrierpunkt 
der beiden Flüssigkeiten bestimmen. Es ist eine aus der Physik bekannte Thatsache, dass die Gefrierpunkterniedrigung im Verhältnisse zur molecularen Concentration der Flüssigkeit, bezw. mit der Zahl der in der Flüssigkeit vorhandenen Molecule steht. Die Gefrierpunkterniedrigung einer Flüssigkeit ist umso grösser, je mehr gelöste Molecule dieselbe enthält und vice versa. Wenn eine Niere daher gesund ist, so hat sie die Fähigkeit, zwischen dem Blute und dem Harn eine gewisse osmotische Druckdifferenz, bezw. eine Differenz in der molecularen Concentration hervorzubringen, welche in Zahlen ausgedrückt werden kann, indem wir die Gefrierpunktserniedrigung der Flïssigkeiten bestimmen, welche unter normalen Verhältnissen mit Bezug auf das Blut $\delta=0,56^{\circ}$, auf den Harn $\Delta=$ 1,2-2,2 " ausmacht. Ist die Niere krankhaft verändert, so vermag sie nicht diese osmotische Druckdifferenz zwischen Blut und Harn hervorzubringen, im Blute bleiben Molecule zurück, was sich darin äussert, dass die Gefrierpunktserniedrigung des Blutes grösser, die des Harns geringer wird. Wenn wir den osmotischen Druck des Harns auf Chlornatrium umrechnen, d. h. bestimmen, wie viel Harn zur Erreichung des normalen osmotischen Druckes bei ausschliesslichem CINa-Gehalte täglich ausgeschieden werden müsste, so geht aus Koranyi's Untersuchungen hervor, dass der in 24 Stunden ausgeschiedene Harn eines normalen Menschen einen osmotischen Druck hat, welcher $30-50 \mathrm{~g}$ Kochsalz entspricht. Aus dem $\Delta$ und der Harnmenge berechnen wir die moleculare Diurese (,a") im $\mathrm{Cl} \mathrm{Na}$ Aequivalente ausgedrückt.

Die Technik dieses Untersuchungsverfahrens ist eine ausserordentlich einfache; mit Hülfe des Beckmann'sehen Thermometers wird die Gefrierpunktserniedrigung von $20 \mathrm{~cm}$ Harn und der Gefrierpunkt von Blut festzustellen gesucht; Blut wird am einfachsten durch Venaesection oder auch Venaepunction entnommen, es ist jedoch nothwendig, nachträglich Oxygen durchzuleiten, um die in demselben enthaltene Kohlensäure auszutreiben; ferner muss auch die 24 stündige Harnmenge Schlüsse werden. Diese Daten genügen, um aus denselben genaue bestimmt zu ziehen. Durch gütige Erlaubniss des Herrn Professor Dollinger habe ich Gelegenheit gehabt, auf der I. chirurgischen Klinik 12 Nierenkranke zu untersuchen. Ich ging in diesen Fällen so vor, dass ich in die krankhaft erscheinende Niere den Ureterkatheter einführte und den Harn dieser Niere sammelte; der Harn der anderseitigen Niere, der gleichfalls gesammelt wurde, entleerte sich durch die Blase; entweder mit Hülfe eines Verweilkatheters oder in der Weise, dass Pat. denselben von Zeit zu Zeit selbst absetzte. Die Harnleiter- 
katheterisation wurde zumeist mit dem Nitze'schen Uretercystoskop, einmal mit dem Albarran'schen Instrumente unter Anästhesie mit 2 proc. Cocainlösung vorgenommen.

I. Fall. Frau M. L., 30 Jahre alt, aufgenommen am 17. October 1899. Gestorben am 20. Januar 1900. - Tuberculosis renalis. Nephrotomie.

Die erste Untersuchung erfolgte am 17. October, wo sich nach Katheterisation der rechten Niere, auf einmal $46 \mathrm{ccm}^{3}$ dichten, milchartig weissen, durchsichtigen Harns entleerte, also renale Retention bestanden hatte.

Harnmenge Spec. Gew. Reaction $A \quad \delta \quad$ a

Rechte Niere nach 12 Stdn. $280 \mathrm{ccm}^{3} 1010 \quad$ alkal. 0.440 .572 .006 Linke " " $12,390 \mathrm{ccm}^{3} 1022$ blutig sauer 1.370 .578 .71

Die chemische Untersuchung des Harns:

Rechte Niere $\begin{aligned} \mathrm{NaCl} & =0.396 \% \\ \mathrm{~N} & =0.17 \%\end{aligned}$ Linke Niere $\mathrm{NaCl}=0.47 \%$

Methylenblauntersuchung:

Rechte Niere:

Linke Niere:

Erscheinen: nach $3 / 4$ Stunden

Ablauf: continuirlich

Dauer: 70 Stunden

nach 1 Stunde

continuirlich

Intensität: Bis zur 5er Farbenscala 7 Farbenscala

Anderthalb Monate nach der Untersuchung am 1. December 1899 Nephrotomie, welche die Diagnose auf Nierentuberculose bestätigte, am 16. December zeigte der Harn der andern Niere $\Delta=0.80$; Pat. starb am 20. Januar 1900 unter Erscheinungen allgemeiner Tuberculose, und die Section ergab, dass auch die linke Niere klein und an einer Stelle eingesunken ist; entsprechend dieser Einsenkung ist ein Kelch erweitert, in dem der Niere zugelegenen Theile käsig infiltrirt, welche Infiltration sich anch auf das Nierengewebe fortsetzt, so dass sich an dieser Stelle ein haselnussgrosser Herd befindet.

II. Fall. Frau T. T., 38 Jahre alt, aufgenommen am 16. Januar 1900, entlassen am 20. April. -- Tuberculosis renalis bilateralis.

Der linke Ureter wurde zu wiederholten Malen katheterisirt. Harnmenge Spec. Gew. Reaction $A \delta$

Linke Niere 24 Stdn. $880 \mathrm{ccm}^{3} \begin{array}{ccccc}1008 & \text { alkalisch } & 0.52 & 0.57 & 5.09\end{array}$ Rechte " $24,1030 \mathrm{ccm}^{3} \quad 1013 \quad \mathrm{amph}$. $0.70 \quad 0.57 \quad 11.07$

Chemische Untersuchung des Harns:

Linke Niere:

$\mathrm{NaCl}=0.222 \%$

$\mathrm{N}=0.128 \%$

Rechte Niere:

$\mathrm{NaCl}=0.304 \%$

$\mathrm{N}=0.37$

Methylenblauntersuchnng:

Linke Niere:

Erscheinen: nach 1 Stunde

Ablauf: intermittirend

Dauer: 66 Stunden

Intensität: bis zur 5er Farbenscala
Rechte Niere:

nach 1 Stunde

intermittirend

56 Stunden

9er Farbenscala 
III. Fall. Frau W. F., 19 Jahre alt, aufgenommen am 25. Januar 1900, entlassen am 2. März 1900. - Linksseitig Pyonephrose.

Durch den Ureterkatheter entleert sich reiner Eiter.

Harnmenge Spec. Gew. Reaction $4 \quad \delta \quad$ a

Linke Niere reiner Eiter -

$\begin{array}{lllllll}\text { Rechte } " 440 & \mathrm{~cm}^{3} & 1030 & \text { saver } & 1.96 & 0.56 & 14.06\end{array}$

Methylenblauntersuchung nach der Nephrotomie.

In dem durch die Blase sich entleerenden Harn

$\left\{\begin{array}{l}\text { Erscheinen: nach } 1 \text { Stunde } \\ \text { Ablauf: continuirlich } \\ \text { Dauer: 48 Stunden } \\ \text { Intensität: bis zur 12er Farbenscala }\end{array}\right.$

Prof. Dollinger hat in diesem Falle die secundäre Nephrektomie ausgeführt.

IV. Fall. M. F., 36 Jahre alt, Mann, aufgenommen am 2. März 1900, entlassen am 27. Juni. - Linksseitige Pyelitis calculosa. Harnmenge Spec. Gew. Reaction $A \quad \delta$ a

Linke Niere $=\begin{array}{llllll}\text { reiner Eiter } & - & - & 0.70 & 0.56 & -\end{array}$ Rechte $\#=1000 \mathrm{ccm} \quad-\quad$ sauer $\quad \begin{array}{llll}1.52 & 0.56 & 7.7\end{array}$

Nach der Nephrotomie entleeren sich durch den Katheter $5 \mathrm{ccm}$ Harn $\Delta=0.48$.

In diesem Falle lag der Ureterkatheter vom 5. bis 31. März, nach erfolgtem Wechsel vom 31. März bis 18. April insgesammt 44 Tage ständig im Ureter, hierdurch wurde eine vollkommene Drainage des Nierenbeckens erreicht. Inzwischen wurde die Nephrotomie vorgenommen und hernach das Nierenbecken durch den Katheter mit Borlösung durchgespült. Derzeit fühlt sich Patient mit seiner Nierenfistel wohl und gelangt zur secundären Nierenexstirpation.

V. Fall. Sch. F., 38. Jahre alt, Mann, aufgenommen am 15. Januar 1900, gestorben am 12. März 1900. Carcinom der rechten Niere. Harnmenge Spee. Gew. Reaction $\Delta \quad \delta \quad$ a

Rechte Niere 12 Stdn. $8 \mathrm{ccm} \quad$ - $\quad$ sauer $0.68 \quad 0.56 \quad 0.8$ Linke " 12 " $335 \mathrm{ccm} \quad$ - $\quad$ - $\begin{array}{llll}1.41 & 0.56 & \mathbf{7 . 7}\end{array}$

Durch den zu wiederholten Malen eingeführten Katheter entleert sich nur wenig Harn. Bei der durch Herrn Dollinger vorgenommenen Nephrektomie stellt sich heraus, dass Mitte und Hilus der rechten Niere von einer grossen carcinomatösen Infiltration eingenommen werden, und auch andere Partieen der Niere zerstreut liegende kleinere und grössere Knoten anfweisen, zwischen den Knoten ist kaum normales Gewebe vorhanden; was unter dem Mikroskope noch als normales Nierengewebe zu erkennen ist, von dem befindet sich ein grosser Theil gleichfalls in infiltrirter hämorrhagischer Nekrose.

Patient überlebte die Operation noch einen Monat, wo er unter Erscheinungen von Krebskachexie starb. Die Obduction ergab, dass die zweite Niere gesund war, in den retroperitonealen und peribronchialen Drüsen grosse Carcinommetastasen. 
VI. Fall. Frau H. L., 41 Jahre alt, aufgenommen am 20. September 1899, entlassen am 12. Nov. 1899. - Wahrsebeinlich Nephritis interstitialis, Ren mobilis.

Beide Ureteren werden auf einmal katheterisirt.

Harnmenge Spec. Gew. Reaction $\Delta \quad \delta \quad a$

Rechte Niere 12 Stdn. $670 \mathrm{ccm} \quad 1010$ alkaliseh $0.42 \quad 0.56 \quad 4.5$

Linke $" 12 \quad \# \quad 580 \mathrm{ccm} \quad 1012 \quad$ sauer $\quad 0.69 \quad 0.56 \quad 6.5$

Gesammtenge während 12 Stunden $690 \mathrm{ccm}$.

Chemische Untersuchung des Harns:

Rechte Niere:

Linke Niere:

$\mathrm{NaCl}=0.269 \%$

$\mathrm{N}=0.24 \%$

$\mathrm{NaCl}=0.315 \%$

$\mathrm{N}=0.61 \%$

Die Harnmenge ist gross, von niedrigem specifischem Gewichte. Eiweissgehalt 5 pro Mille. Die paroxysmenweise auftretenden Schmerzen, derentwegen Pat. auf die I. chirurgische Klinik gelangte, waren durch ihre rechtseitige Wanderniere, bezw. die intermittirende Hydronephrose verursacht. Das Aufhören der Schmerzen am Ende solcher Anfälle war stets von Entleerung reichlichen Harns gefolgt, Pat. verliess ohne Operation die Klinik.

VII. Fall. Frau G. M., 23 Jahre alt, aufgenommen am 8. Juli 1899, entlassen am 10. Februar 1900. - Nierenfistel nach rechtsseitiger Nephrotomie.

Der Harn wird mittelst Katheterisation des rechten Ureters, sowie durch die Fistel gesammelt. Der Harn der anderen Niere entleert sich durch die Blase.

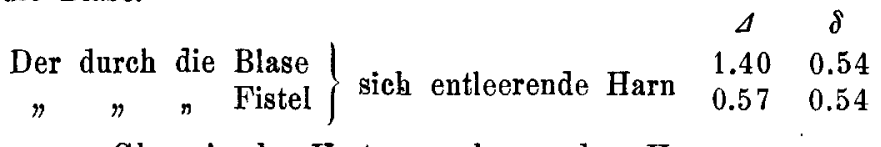

Chemische Untersuchung des Harns:

In dem durch die Fistel sich entleerenden Harn ist $\mathrm{NaCl}=0.35 \%$, $\mathrm{N}=0.38$.

$$
\text { Methyleublauntersuchung: }
$$

Rechte Niere:

Erscheinen: nach $11 / 2$ Stunden

Ablauf : continuirlich

Daner: 48 Stunden

Intensität: 5er Farbenscala
Linke Niere:

nach 1 Stunde

continuirlich

5 Tage

13 er Farbenscala

Herr Prof. Dollinger nahm die secundäre Nephrektomie vor, nach welcher Pat. geheilt entlassen wird.

VIII. Fall. Frau P. F., 51 Jahre alt, aufgenommen am 3. April 1899, entlassen am 6. November 1899. - Nach Nephrektomie zurtickgebliebene gesunde Niere.

Beim Pat. hat Prof. Dollinger bereits fruher die Nephrektomie vorgenommen, er verblieb nur wegen seiner tuberculösen Blasengeschwüre in der Klinik. $\Delta=1.85 \delta=0.56$. 
Methylenblauntersuchung:

Erscheinen: nach $11 / 2$ Stunden Ablauf: continuirlich
Dauer: 84 Stunden

Intensität: $14 \mathrm{er}$ Farbenscala.

IX. Fall. F. K., 22 Jahre alt, aufgenommen am 2. November 1899, entlassen am 21. November 1899. - Gesunde Nieren.

Pat. hatte paroxysmenweise auftretende Schmerzen in der rechten Nierengegend, in der letzten Zeit ging Nierensand mit dem Harn ab.

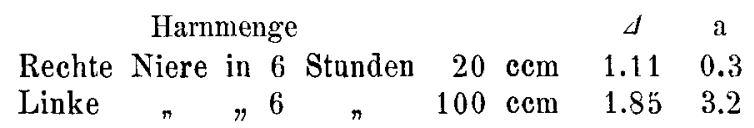

Methylenblauntersuchung des Gesammtharns:

Erscheinen: nach $11 / 2$ Stunden Dauer: 52 Stunden

Ablauf : continuirlich

Intensität: 24er Farbenscala.

Pat. verlässt die Klinik, steht seit einem halben Jahre in Beobachtung, fühlt sich vollkommen wohl.

X. Fall. X. J., 20 Jahre alt, Mann. - Gesunde Nieren.

In der rechten Lumbalgegend ein grosser Abscess füblbar. Spondylitis dorsalis, rechtsseitiger Ureterkatheterismus.

Harnmenge Spec. Gew. Reaction $\Delta \quad \delta \quad$ a

$\begin{array}{lllllll}\text { Rechte Niere in } 24 \text { Stdn. } \quad 5 \mathrm{cem} \quad- & \text { sauer } & 1.91 & 0.56 & 1.5\end{array}$ Linke " $, 24 \quad, 430 \mathrm{cem} \quad 1019 \quad \% \quad 1.30 \quad 0.56 \quad 9.1$

Die Operation bestätigt das Vorhandensein eines von der Spondylitis herrührenden praeperitonealen Abscesses.

XI. Fall. X. Y., 19 Jahre alt, IIann. - Gesunde Nieren.

Pat. klagt uber seit langer Zeit bestehende Schmerzen auf der linken Seite. Spondylitis dorsalis, linksseitiger Ureterkatheterismus.

Harnmenge Spec. Gew. Reaction $\Delta \quad \delta \quad$ a

Linke Niere in 10 Stunden $144 \mathrm{com} 1024$ alkalisch. $1.86-2.9$ Rechte " "10 " $90 \mathrm{ccm} 1020$ amph. $1.31-1.9$

XII. Fall. Frau X. Y., 16 Jahre alt. - Gesunde Nieren.

Seit einigen Monaten Schmerzen in der linken Nierengegend, ein grosser Abscess daselbst. Tuberculinreaction positiv, Spondylitis dorsalis, linksseitiger Ureterkatheterismus.

$$
\begin{array}{lllcc}
\multicolumn{3}{c}{\text { Harnmenge }} & \Delta & \text { a } \\
\text { Linke Niere } & 970 & \mathrm{ccm} & 1.10 & 19.3 \\
\text { Rechte } " & 650 \mathrm{ccm} & 1.46 & 15.4
\end{array}
$$

Der Inhalt des grossen Abscesses wird von Prof. Dollinger mittelst dünnen Troicarts abgelassen, in die Höhle 10 Proc Jodoformglycerinemulsion eingespritzt. Pat. erhält ein Spondylitismieder.

Aus diesen Fällen ist ersichtlich, welche wichtige Rolle dem Ureterenkatheterismus in der Diagnostik der chirurgischen Nierenkrankheiten zufällt, sowohl vom Standpunkte der Entscheidung, ob eine kranke Niere vorhanden ist, als auch dessen, ob die andere ge- 
sund ist. Die Kenntniss dieses Umstandes ist zwecks Wahl des vorzunehmenden Eingriffes unbedingt nothwendig. Der Ureterenkatheterismus ist neben den beschriebenen Untersuchungsmethoden nur als ein Hülfseingriff zu betrachten, um den Harn jeder einzelnen Niere zur weiteren Prüfung gesondert auffangen zu können.

Die chemische Untersuchung des Harns hat selbst dann nicht viel Erfolg, wenn das Secret der einzelnen Niere gesondert gepriift wird, und zwar deshalb nicht, weil die erhaltenen Werthe auch unter normalen Verhältnissen derart schwanken, dass keine feineren Schlüsse abgeleitet werden können, nur die gröberen Abweichungen von der Norm sind es, welche diagnostisch verwerthbar sind; so lassen sich nur aus dem Vergleiche der aus dem Harn der zwei Nieren erhaltenen Zahlenangaben Schlüsse von relativem Werthe ziehen.

Die Prüfung mit Methylenblau gestattet schon mehr zu folgern; als Zeichen compensatorischer Hypertrophie ist der protrahirte Ablauf der Secretion in den Fällen VII und VIII sehr schön ersichtlich, wo nach Entfernung der einen Niere die andere ihre Function übernommen hat. Dieses Verfahren ist jedoch langwierig, erst muss eine Farbenscala angelegt, der Harn des Kranken stündlich gesammelt, dann diese Harne gesondert untersucht werden und zwar erst das in oxydirter Form erschienene Methylenblau, dann das im aufgekochten und angesäuerten Harn zum Vorschein kommende Chromogen; die Untersuchungsergebnisse sind trotzdem nicht ganz genaue, selbst dann nicht, wenn wir nicht durch Vergleichung mit der Farbenscala, sondern die ausgeschiedenen Methylenblaumengen bestimmen, welches Verfahren jedoch ein ausserordentlich langwieriges ist.

Die genauesten Resultate ergiebt unter allen Umständen das Koranyi'sche Gefrierungsverfahren; bei chirurgischen Nierenkrankheiten ist es jedoch ein unbedingtes Erforderniss, dasselbe gleichzeitig mit dem Ureterkatheterismus vorzunehmen. Denn der Gefrierpunkt des Gesammtharns kann auch bei einseitiger Nierenerkrankung normal sein, wenn die andere Niere gesund ist. Derselbe kann sogar auch dann normal sein, wenn beide Nieren krank sind, wo dann der normale Gefrierpunkt des Blutes darauf hinweist, dass die zwei pathologischen Nieren die Functionen versehen können. Wenn in solchen Fä̈len nicht der Harn der einzelnen Niere gesondert geprüft wird, so tritt nach Entfernung der einen Niere Insufficienz ein und der Patient geht früher oder später zu Grunde. Nur in einem Falle kann aus dem Gesammtharn auf den Zustand der Nieren geschlossen werden, nämlich dann, wenn die Gefrierpunktserniedrigung des Blutes grösser als normal, der Moleculargehalt des Blutes daher grösser, also renale 
Insuffieienz vorhanden ist, welehe ron einer Erkrankung der Nieren oder z. B. grossen $\Lambda$ bdominaltumoren herrühren kann, welche Circulationsstïrungen veranlassen.

Die Gefrierpunkte des Harns gestatten übrigens nur auf dem functionellen, nicht aber den anatomischen Zustand der Nieren einen Rïckschluss; deshalb, weil die Gefrierpunktserniedrigung des Harns von der Function der Niere abhängt, diese Erniedrigung unter normalen Verhältnissen schon grossen Schwankungen unterworfen, welche wiederum beispielsweise von der getrunkenen Wassermenge abhängig ist; es wäre daher wünschenswerth, die Methode dahin auszubauen, dass diese Schwankungen auf ein Minimum herabgesetzt und auch die anatomischen Veränderungen der Nieren diagnosticirt werden künnen.

Die Bestimmung des Gefrierpunktes des Blutes ist in jedem einzelnen Falle sehr wichtig, einerseits um zu erfahren, ob eine Niereninsufficienz vorliegt, andererseits vom Standpunkte der Prognose aus, welche wir bei grösserer Gefrierpunktserniedrigung für weniger günstig als bei normalem aufstellen müssen. Auch Albarran hat derartige Untersuchungen vorgenommen, in 9 Fällen jedoch die Prüfung des Gefrierpunktes des Blutes unterlassen, und gerade dieser Theil der Untersuchung ist es, wodurch dieselbe vollkommener ist als die Prüfung der Methylenblauausscheidung, mit Hülfe welcher keine solch genaue Diagnose und Prognose festgestellt werden kann. Kümmel gelangt zum Resultate, dass nach Bestimmung der Gefrierpunktserniedrigung des Blutes der der Harne nur dann erforderlich ist, wenn uns das Blut keine Aufklärung gewährt. Wir wissen jedoch - wie erwähnt - dass in gewissen Fällen aus dem Blute keinerlei Schlüsse abgeleitet werden können, hingegen aus dem gesondert aufgefangenen Harn, wie dies auch aus meinen Fällen zu ersehen ist. Wichtig ist daher nicht nur die Bestimmung der Gefrierpunktserniedrigung des Blutes, sondern auch der gesondertaufgefangenen Harne. Die Abnalme der Gefrierpunktserniedrigung des Harns steht beiläufig im Verhältniss zum Grade der Nierenerkrankung; wie beispielsweise Fall VII zeigt, wo der Gefrierpunkt des aus der zu Grunde gegangenen Niere stammenden Harns sich kaum vom Gefrierpunkt des Blutes unterscheidet, wo die Niere absolut nicht im Stande ist, eine osmotische Druckdifferenz zwischen Blut und Harn hervorzubringen, die ausgeschiedene Flüssigkeit eigentlich nur ein Transsudat ist. Wichtig ist auch noch der Umstand, dass die Kryoskopie technisch ein solch einfaches Verfahren darstellt, welches von jedermann in einigen Minuten ausgeführt werden kann, nicht so lang- 
wierig ist, als die chemische Untersuchung oder die Bestimmung des ausgeschiedenen Methylenblau.

Die moleculare Diurese können wir durch Multiplication der Tagesmenge der Harne mit 1 und Dividiren durch 073, dem Gefrierpunkt der Kochsalzlösung, berechnen, wo wir dann dieselbe in $\mathrm{NaCl}$ Aequivalenten ausgedrückt erhalten. Aus dieser Zahl können wir gleichfalls Schlüsse von, wenn auch nicht absolutem, so doch relativem Werthe ableiten, indem wir sehen können, um wie viel die durch die kranke Niere producirte moleculare Diurese kleiner ist als die der gesunden Seite.

Die Berechnung der molecularen Diurese hat dann den grössten Werth, wenn die Harnbestandtheile in viel Wasser gelöst entleert werden, wenn die moleculäre Concentration, also $I$ klein ist, während bei Berechnung der moleculären Diurese die Abweichung von der Norm, wie Fall VI zeigt, geringer erscheint.

In den von mir untersuchten Fällen können wir sehr gut den Werth dieser verschiedenen Untersuchungsmethoden vergleichen, wo dieselben in ein und demselben Falle parallel geschahen. Wir können ersehen, um wie viel genauere diagnostische und prognostische Fingerzeige das Koranyi'sche Verfahren liefert als die übrigen, jedoch bloss dann, wenn der Harn der Nieren gesondert untersucht wird.

Der Ureterenkatheterismus ist bei sorgfältiger Ausführung mit sterilen Instrumenten überhaupt kein gefährlicher Eingriff; der Katheter kann lange Zeit, wie in Fall IV 44 Tage, liegen gelassen werden, ohne dem Patienten Unannehmlichkeiten zu bereiten; während dieser Zeit kann der Harn öfters untersucht, der Grad der Veränderungen festgestellt und eventuell jene neueren conservativen Behandlungsmethoden ausgeführt werden, welche darauf hinzielen, per vias naturales, ohne Anlegung einer Wunde, gewisse Erkrankungen der Niere in Heilung überzuführen und die Niere zu erhalten.

Zum Schlusse ist es mir eine angenehme Pflicht, meinem sehr verehrten Chef, Herrn Prof. Dollinger meinen aufrichtigsten Dank auszudrücken für sein bereitwilligstes Entgegenkommen und die vielfachen guten Fingerzeige, die ich ihm noch besonders verdanke. 